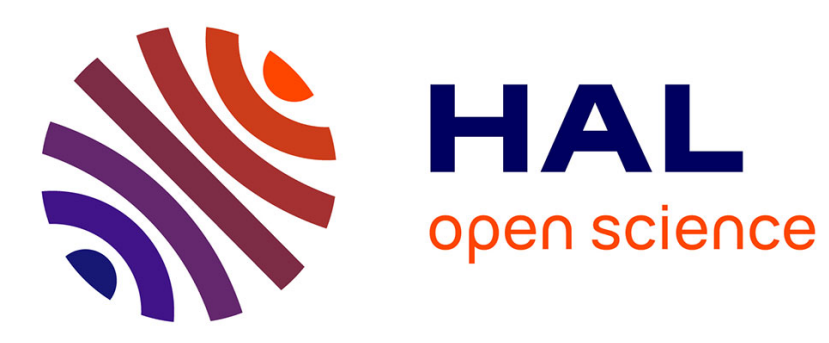

\title{
Use of a micromechanical approach to understand the mechanical behavior of solid propellants
}

Foucault de Francqueville, Julie Diani, Pierre Gilormini, Aude Vandenbroucke

\section{To cite this version:}

Foucault de Francqueville, Julie Diani, Pierre Gilormini, Aude Vandenbroucke. Use of a micromechanical approach to understand the mechanical behavior of solid propellants. Mechanics of Materials, 2021, 153, pp.1-9. 10.1016/j.mechmat.2020.103656 . hal-03040246

\section{HAL Id: hal-03040246 https://hal.science/hal-03040246}

Submitted on 4 Mar 2021

HAL is a multi-disciplinary open access archive for the deposit and dissemination of scientific research documents, whether they are published or not. The documents may come from teaching and research institutions in France or abroad, or from public or private research centers.
L'archive ouverte pluridisciplinaire HAL, est destinée au dépôt et à la diffusion de documents scientifiques de niveau recherche, publiés ou non, émanant des établissements d'enseignement et de recherche français ou étrangers, des laboratoires publics ou privés. 


\title{
Use of a micromechanical approach to understand the mechanical behavior of solid propellants
}

\author{
Foucault de Francqueville ${ }^{\mathrm{a}, \mathrm{b}}$, Julie Diani ${ }^{\mathrm{a}, *}$, Pierre Gilormini ${ }^{\mathrm{c}}$, Aude Vandenbroucke ${ }^{\mathrm{b}}$ \\ ${ }^{a}$ Laboratoire de Mécanique des Solides, CNRS UMR 7649, École Polytechnique, Institut Polytechnique de Paris, Route de Saclay, 91128, Palaiseau, France \\ ${ }^{\mathrm{b}}$ ArianeGroup, Centre de Recherche Du Bouchet, 9 Rue Lavoisier, 91710, Vert-le-Petit, France \\ ${ }^{c}$ Laboratoire PIMM, ENSAM, CNRS, CNAM, 151 Bd de L'Hôpital, 75013, Paris, France
}

\begin{tabular}{|c|c|}
\hline & A B S T R A C T \\
\hline $\begin{array}{l}\text { Keywords: } \\
\text { Propellants } \\
\text { Micromechanics } \\
\text { Debonding } \\
\text { Damage } \\
\text { Cohesive-zone model }\end{array}$ & $\begin{array}{l}\text { The development of new generations of propellants with better energetic properties may be hampered by un- } \\
\text { satisfactory mechanical behaviors in terms of strength and toughness. A micromechanical approach is adopted to } \\
\text { provide a better understanding of the existing links between the constitutive phase behaviors and the local } \\
\text { damage, and the macroscopic mechanical behavior of these materials. Three model materials have been made } \\
\text { and tested in uniaxial tension. The stress-strain responses were recorded while monitoring their volume changes } \\
\text { that quantify the macroscopic damage. A qualitative description of the local damage was obtained thanks to } \\
\text { scanning electron microscopy images of samples under loading. The micromechanical approach consists in finite } \\
\text { elements analyses on periodic microstructures of non-regular polyhedral particles embedded in a soft matrix. An } \\
\text { original microstructure generation tool has been developed specifically in order to obtain highly filled isotropic } \\
\text { microstructures. Debonding at the matrix/filler interface was taken into account with a cohesive-zone model } \\
\text { (CZM). The impact of the CZM parameters is discussed, in an effort to make the link between the CZM parameters } \\
\text { and how the local damage appears and develops, and between the cohesive behavior and the shape of the } \\
\text { macroscopic stress-stretch responses of the heterogeneous materials. }\end{array}$ \\
\hline
\end{tabular}

\section{Introduction}

Solid propellants are used for anaerobic propulsion of both civil and military launchers. While their combustion is probably the main focus of materials developers, their mechanical behavior remains a major concern to ensure rockets integrity since the presence of damage such as cracks may alter their combustion efficiency and stability (Kumar et al., 1981). Therefore, the development of new propellants with enhanced combustion properties requires to provide materials with similar or better mechanical properties in terms of both strength and toughness.

The strength and toughness of propellants are usually assessed on macroscopic stress-strain responses obtained in uniaxial tension. However, the various shapes of the stress-strain responses that may be obtained are not well linked to the behavior of the different constitutive phases and the damage appearing at the microscale. In such heterogeneous materials, highly filled with micrometric particles, the damage appears as matrix debonding (Oberth and Bruenner, 1965; Cornwell, 1975) around the larger particles (Oberth and Bruenner, 1965; Cornwell and Schapery, 1975; Heuillet, 1992) at the matrix/filler interfaces. In order to model such a damage, finite elements simulations including cohesive zone model (CZM) elements at the particle/matrix interface of heterogeneous microstructures, have been often chosen. While several numerical micromechanical simulations have been proposed for soft matrices reinforced by spherical fillers, with an increasing complexity following the progress of computational capacity (for instance Zhong and Knauss (1997, 2000); Matouš et al., 2006; Inglis et al., 2007, Gilormini et al., 2017), simulations dealing with more realistic polyhedral particles are scarce. They are recent and generally run in 2D (Barua et al., 2012; Keyhani et al., 2019; Dai et al., 2019; Huang et al., 2020). In order to account for a very large fraction of fillers, 2D grains are considered and the binder appears as seals between the grains. This representation is not in good agreement with the microstructures that have been observed by scanning electron microscopy (Li et al., 2018). Simulations considering 3D polyhedral particles randomly dispersed in a matrix (Williams et al., 2012; Weng et al., 2019) are dealing with metal matrices, which behaviors differ significantly from the soft hyperelastic binder used in propellants.

The present contribution aims at using a numerical micromechanical

\footnotetext{
* Corresponding author.

E-mail address: julie.diani@polytechnique.edu (J. Diani).
} 
modeling approach to describe the link between the local mechanical behaviors and damage, and the macroscopic mechanical behavior of actual model propellants. For this purpose, original experimental data have been obtained on three types of hydroxyl-terminated polybutadiene (HTPB) model propellants formulated by ArianeGroup, which show notably different mechanical behaviors when tested in uniaxial tension. Scanning electron microscopy (SEM) observations have given access to the local damage occurring upon loading for the three materials, without providing explanations for the strong difference of behaviors between the materials. To provide a rationale on these experimental results, finite element simulations have been run on representative volume elements made of polyhedral particles randomly dispersed in a soft hyperelastic matrix with an account of possible matrix/filler interfacial damage thanks to CZM elements. A specific effort has been carried on the generation of representative microstructures with a relatively high volume fraction of fillers (up to 55\%), randomly dispersed to avoid undesired anisotropy (de Francqueville et al., 2020b). Fillers are non regular polyhedra randomly translated and rotated to avoid possible numerical bias in damage appearance due to similar parallel edges facing each others. Such a micromechanical approach favors a thoughtful understanding of the relationship between the macroscopic behavior of the composite and its local damage (de Francqueville et al., 2020a). It should provide with insightful explanations of the different macroscopic behaviors witnessed on the actual materials.

The next section presents the actual model materials. Their mechanical behaviors have been characterized in monotonic uniaxial tension. The Young moduli defining the initial linear responses are extracted, and the finite strain stress-strain responses are displayed until break. The samples volume changes upon loading, which outline the amount of damage, have been recorded at the same time as the stressstrain responses. Images of the micro-scale matrix/filler debonding are shown and discussed. Section 3 presents the computational framework for the micromechanical calculations using finite element analyses. A first discussion on the linear elastic results is offered including the comparison with mean field homogeneization calculations obtained with the generalized self-consistent model, classically adopted for matrices filled with spherical particles.

\section{HTPB propellants characterization}

\subsection{Materials}

Three materials, based on a HPBT matrix filled with different types of particles, were made at ArianeGroup. For safety reasons, an inert matrix was chosen. In order to be representative of actual solid propellants, ammonium perchlorate (AP) fillers were added to the matrix or to a premix containing the matrix filled with $20 \%$ in volume of fine aluminium particles of $\approx 10 \mu \mathrm{m}$ diameters that are often used as an extra oxidizer. The new generation of energetic particles were also considered with hexogen (RDX), added to the plain inert matrix. In order to deal with materials easy to model and not hold all the complexity of industrial propellants, the granulometry of the fillers has been reduced to a range of 275-300 $\mu \mathrm{m}$. The material denominations and compositions are listed in Table 1. Materials have been elaborated following as closely as possible an industrial process. Avoiding air bubbles, fillers sedimentation or decantation, the obtained volume fraction of fillers depends on the viscosity of the matrix premix. Propellant chemical additives usually introduced, have been added also. At the end of the curing process, dogbone shape samples with a working length of $80 \mathrm{~mm}$ were cut and glued with Loctite Flexcomet PU 15 to aluminum jaws for mechanical testing. Each sample has been kept for 15 day at $20 \mathrm{C}$ in a controlled atmosphere with bellow $10 \%$ of relative humidity.
Table 1

Compositions and denominations of the propellant-like composites.

\begin{tabular}{lllll}
\hline $\begin{array}{l}\text { Material } \\
\text { denomination }\end{array}$ & Matrix & Fillers & $\begin{array}{l}\text { volume } \\
\text { fraction of } \\
\text { fillers } \\
f_{\text {vol }}(\%)\end{array}$ & $\begin{array}{l}\text { Aluminium } \\
\text { powder } \\
\text { vol. fraction in } \\
\text { matrix (\%)) }\end{array}$ \\
\hline AP_al & Inert & $\begin{array}{l}\text { ammonium } \\
\text { perchlorate } \\
\text { ammonium } \\
\text { perchlorate } \\
\text { RDX }\end{array}$ & 58 & 20 \\
RP & Inert & Inert & 53 & $\varnothing$ \\
\hline
\end{tabular}

\subsection{Mechanical responses to monotonic uniaxial tensile tests}

The binder and propellants were submitted to uniaxial tensile tests at 20C constant temperature and $5 \mathrm{~mm} / \mathrm{min}$ constant crosshead speed. Composite samples were tested on an Instron 5967 tensile machine equipped with a Farris dilatometer (Farris, 1964). The Farris setup consists in an airtight chamber with pressure monitoring. From this setup, one may record the sample volume changes upon uniaxial tensile test. Fig. 1a shows the binder stress-strain response well reproduced by the Ogden hyperelastic model (Ogden, 1972) with one pair of parameters ( $\mu=0.11 \mathrm{MPa}, \alpha=2.25$ ) only. Fig. 1b displays the propellants stress-strain responses and dilatations recorded during the uniaxial tensile tests.

Focusing on the linear part of the stress-strain responses at small strain, one may extract the Young modulus values as shown in Fig. 2, where the dashed lines are the linear approximations of the stress-strain responses of the composites. The Young modulus $E$ of the polymer matrix was determined at $0.33 \mathrm{MPa}$ by linear regression at small strain, which is consistent with the shear modulus $\mu=0.11 \mathrm{MPa}$ displayed by the Ogden fit in Fig. 1a for a quasi-incompressible matrix of Poisson's ratio $v$ close to 0.5 . The values of the Young moduli of the fillers were extracted from the literature, where data obtained by nano-indentation report values of $19.5 \mathrm{GPa}$ for ammonium perchlorate (Lucca et al., 2006) and 16 GPa for RDX (Hudson et al., 2012). A standard Poisson's ratio of 0.25 is assumed for both types of fillers.

At large strain, one notes three rather different mechanical behaviors in terms of shapes and values of strain at break. Nonetheless, for every material, the dilatation is null at the beginning of the loading due to the rubbery matrix very low compressibility, then it increases with the appearance of cavities in the material (as SEM images will show below). The initiation of volume change is concomitant with the material softening witnessed on the stress-strain curve. The material AP_al, which matrix has been premixed with fine aluminium particles, displays a slow softening allowing to reach high level of stress but sudden failure happens early. To the contrary, materials AP and RDX show some strong softening and larger strains at failure. While the onset of volume changes allows determining the onset of the material damage, the similar linear evolutions of the material dilatations cannot explain the different trends in the stress-strain responses. In order to observe the physics of the damage, source of the material softening recorded during the mechanical tests at the macroscopic scale, scanning electron microscopy observations have been carried out on stretched samples.

\subsection{Micro-scale observations of the matrix/filler debonding}

Images were obtained with a Zeiss JSM-6510 microscope with $8 \mathrm{keV}$ beam energy. The composites microstructures are displayed before any applied loading in Fig. 3. The sizes of the particles are similar, they are not spherical but present some facets although their shape ratios are close to one. The geometries of the ammonium perchlorate and RDX fillers appear as similar and cannot explain the differences of behaviors reported in the previous section.

Fig. 4 shows the local damage observed in material AP_al when 


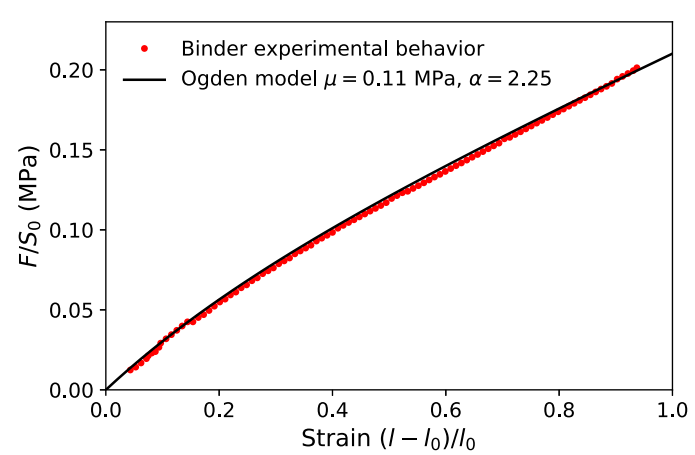

(a)

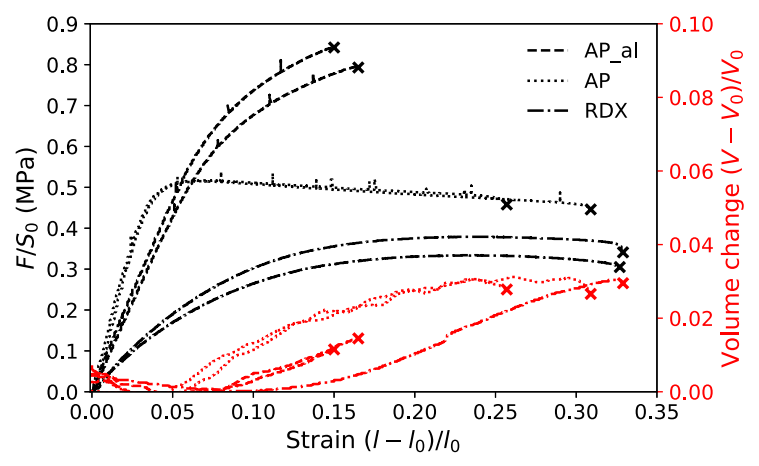

(b)

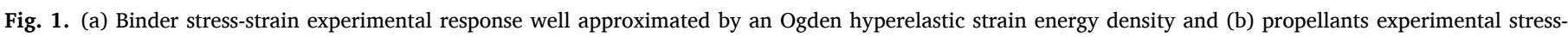
strain responses and volume changes recorded during uniaxial tensile tests carried at 20C constant temperature and constant crosshead speed of 5 mm/min.

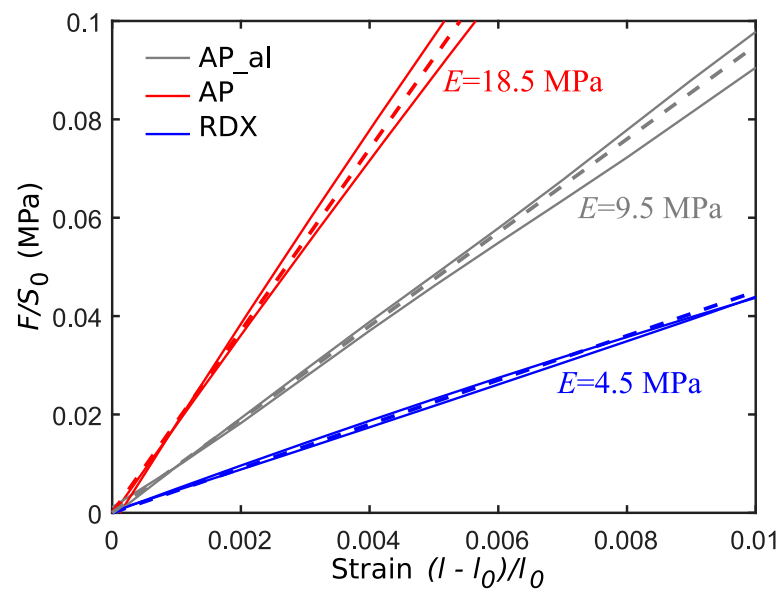

Fig. 2. Estimates of the Young modulus characterizing the small strain linear part of the stress-strain responses displayed in Fig. 1.

vertically stretched at $11 \%$ strain. The global view of the material shows that the damage results from matrix debonding around the particles of ammonium perchlorate. Moreover, the damage is spread across the material without localization. On the zoomed-in view, one notices numerous matrix fibrils around a particle, proving that while the matrix/filler interface is damaged, it is far from being completely broken. This was consistently witnessed for material AP_al.

Fig. 5 displays the local damage state of material AP submitted to a vertical uniaxial stretching of $15 \%$ strain. In the global view of material AP, one notices that the damage seems to develop along lines. Additionally, fine observations reveal that most of the particles on these lines, are completely detached from the matrix.

Finally, Fig. 6 shows the local damage of material RDX recorded for a vertical uniaxial strain of $19 \%$. The global view of the material offers a similar image as composite AP_al, with cavities surrounding particles, randomly dispersed across the microstructure. Polymer fibrils are visible around the particles showing matrix debonding, even though $19 \%$ of strain was reached for this material. Nonetheless, the fibrils coexist with some zone of complete matrix debonding.

The local damage observations seem to classify materials AP_al and $\mathrm{RDX}$ in the same category with damage randomly dispersed across the microstructure, and matrix debonding exhibiting polymer fibrils attached to the particles. Material AP shows some damage localization in terms of matrix debonding developing along lines and complete matrix debonding around the fillers where interfacial damage occurs. Unfortunately, the simple analysis carried out in this section cannot explain the difference between the mechanical responses reported above. Therefore, in the next section, we propose to run finite elements simulations on representative microstructures in order to interpret the macroscopic stress-strain responses that have been obtained experimentally.

\section{Micromechanical simulations}

\subsection{Computational framework}

The composites are modeled by cubic periodic microstructures containing 64 spherical or polyhedral particles filling 55\% of the volume. The microstructure building process starts with spherical particles randomly dispersed in a cubic box thanks to a Lubachevsky and Stillinger algorithm (Lubachevsky and Stillinger, 1990). Such an algorithm may be downloaded at (Torquato, 2006). This provides with spherical

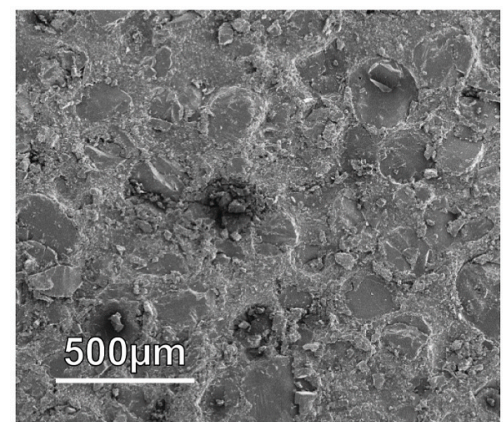

AP_al

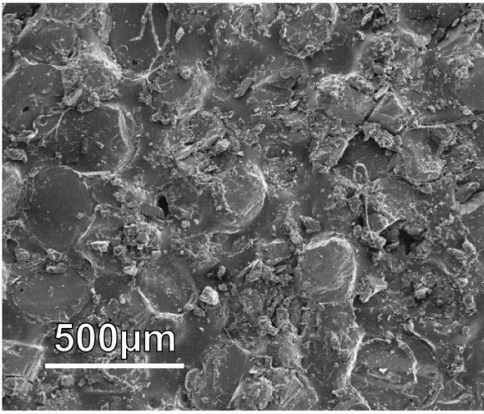

AP

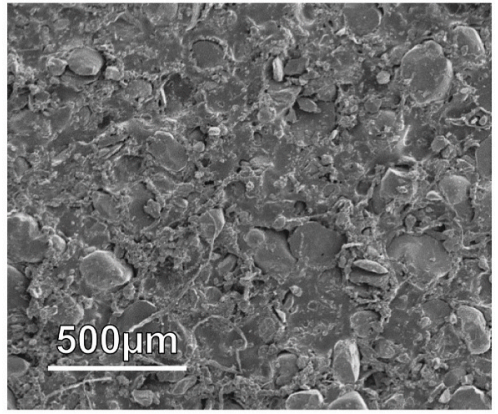

RDX

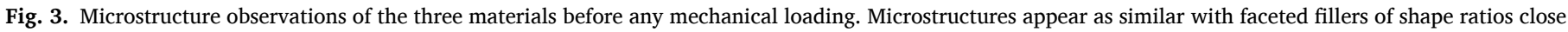
to one. 

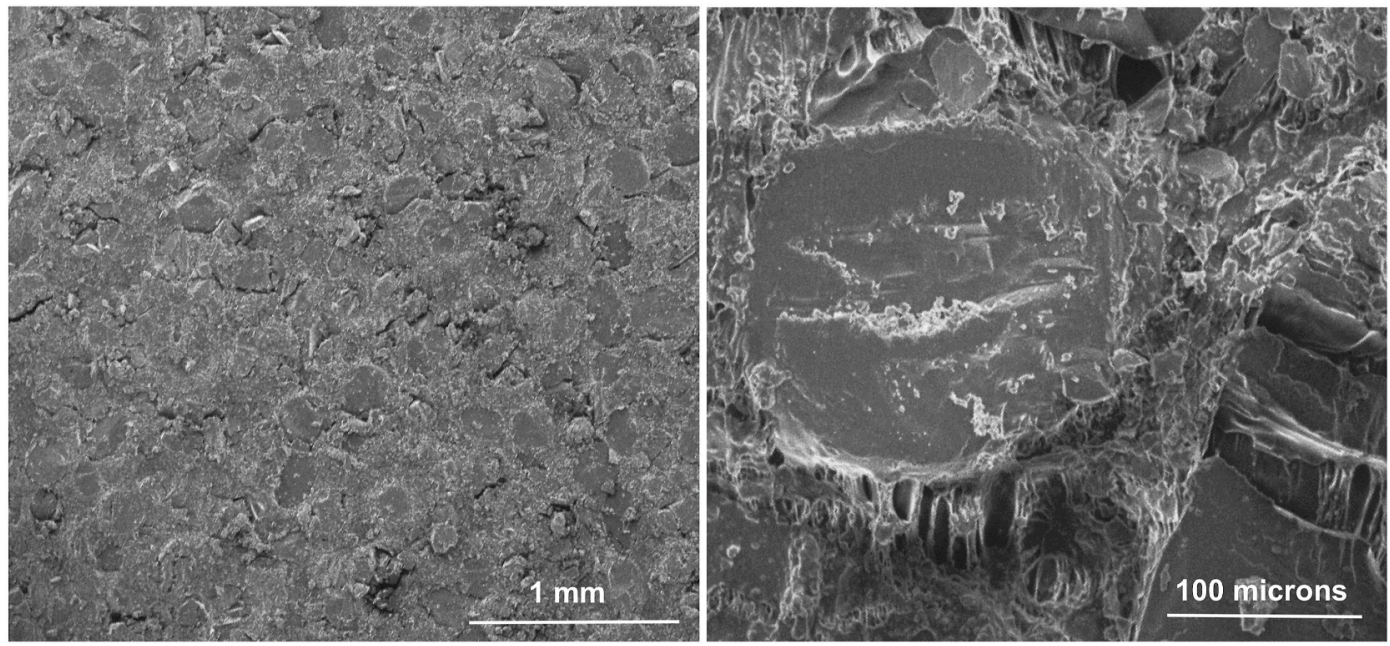

Fig. 4. SEM observations of material AP_al submitted to $11 \%$ strain vertical uniaxial stretching.
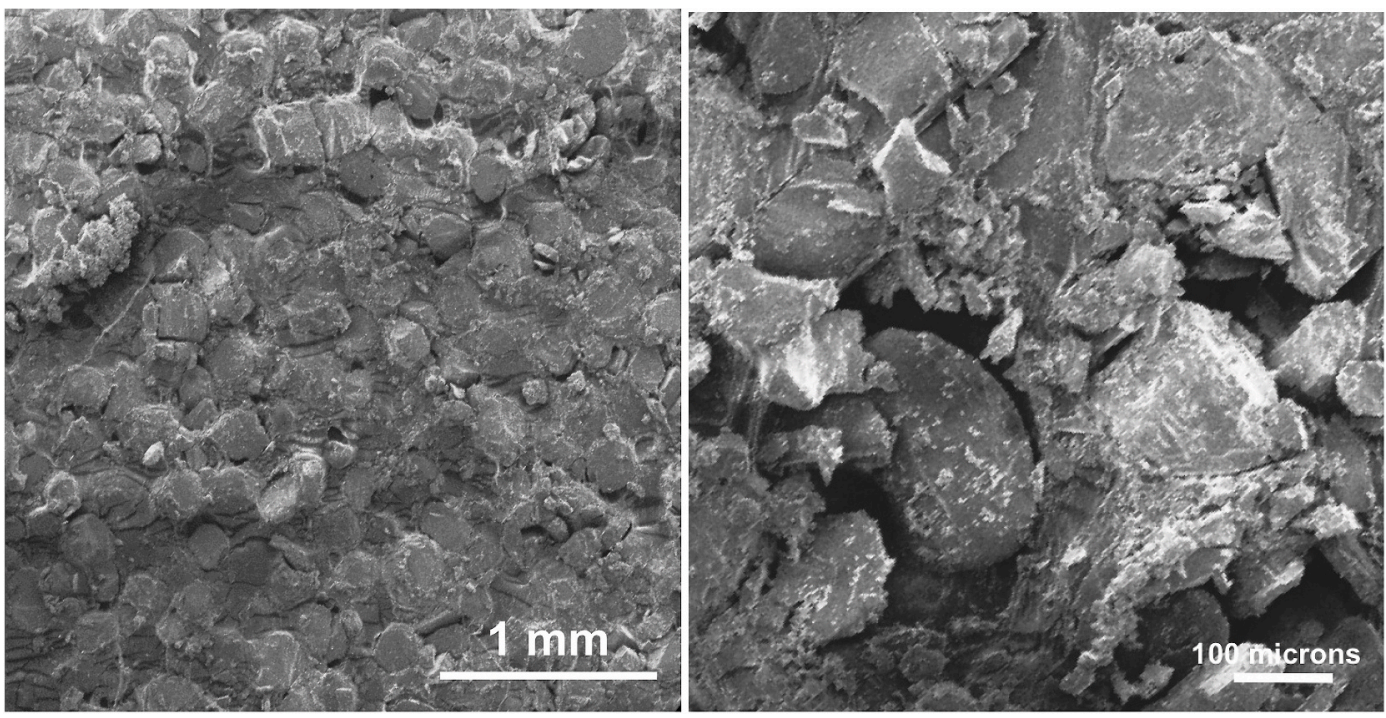

Fig. 5. SEM observations of material AP submitted to $15 \%$ strain vertical uniaxial stretching.
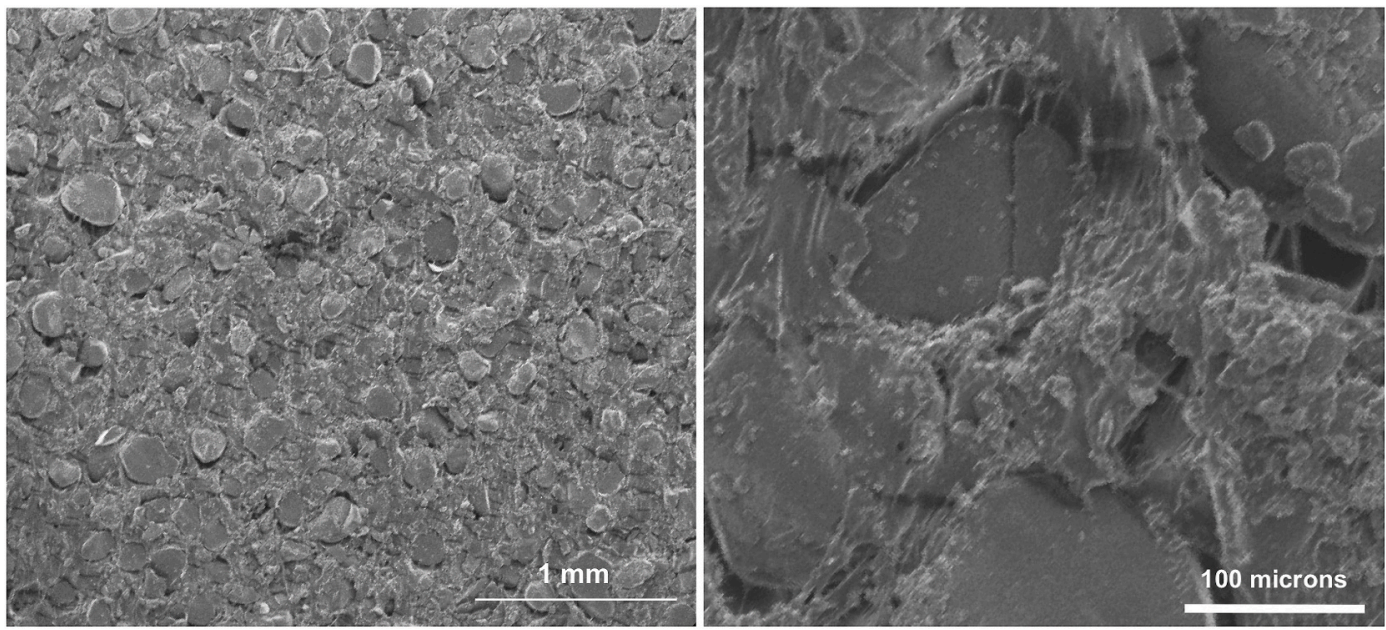

Fig. 6. SEM observations of material RDX submitted to $19 \%$ strain vertical uniaxial stretching. 
particles embedded in a matrix (Fig. 7a). In order to build similar microstructures with non-regular polyhedral particles (Fig. 7b), the centers of the spheres of an isotropic microstructure (de Francqueville et al., 2019) are used as seed points for a Voronoï diagram. The obtained polyhedra are shrinked to obtain particles of the same volumes and the targeted overall volume fraction. Finally, random rotations and translations of the polyhedra have been added in order to avoid regular planes facing each other and obtain a more realistic virtual material. The procedure inspired by Fritzen et al. (2009) is detailed in de Francqueville et al. (2020b). For the finite elements analyses, the meshing was carried out with Netgen software (Schöberl, 1997) and calculations were run on Abaqus (2018). Netgen stands as an efficient tool for periodic meshes listing the equations required by the periodic boundary conditions. Ten-node tetrahedral elements were used for the fillers (C3D10 abaqus elements) and the matrix $(\mathrm{C} 3 \mathrm{D} 10 \mathrm{H}$, due to the close to incompressible behavior). Mesh failures could be obtained when a polyhedron vertex was too close to an edge, face or vertex of any other polyhedron or of the cubic cell. A strategy, inspired by Quey et al. (2011), that creates new tiny faces at the polyhedron vertices creating the difficulties, was applied as one can read in de Francqueville et al. (2020b). Representative meshed microstructures are shown in Fig. 7.

The relatively low number of 64 particles has been proven to be enough for representative periodic cubic cells with spherical particles (Gusev, 1997). In order to confirm its relevance for polyhedral particles, sets of five microstructures with 8, 16, 32, 64 and 128 particles have been generated and submitted to uniaxial tension and shearing along the principal axes of the cubic cells. The values of the homogeneous Young modulus and shear modulus obtained numerically are shown in Fig. 8 which proves that 64 is a good compromise to achieve relevant results without carrying too large meshes. In order to validate the mesh convergence, simulations with up to 2 millions elements were run showing a plateau of convergence once past the million of elements.

As discussed previously in section 2.2, the behavior of the quasiincompressible rubbery matrix is modeled by the Ogden hyperelastic strain energy with a single pair of parameters, $\mu=0.11 \mathrm{MPa}$ and $\alpha=$ 2.25 The particles are assumed linear elastic with a Young modulus according to the literature $16 \mathrm{GPa}$ for the RDX and $19.5 \mathrm{GPa}$ for the AP fillers, and a Poisson's ratio of 0.25 .

In order to account for the matrix/filler debounding, cohesive-zone elements have been added at the matrix/filler interface. For this purpose, a simplified version of the Tvergaard and Hutchinson potentialbased model (Tvergaard and Hutchinson, 1993) has been chosen. An illustration of the local damageable behavior simulated with such elements is displayed in Fig. 9 in the case of purely normal or tangent

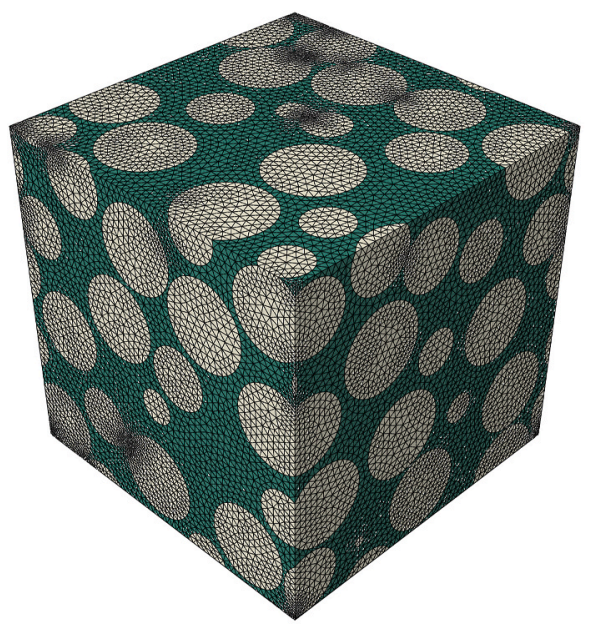

(a) displacement. The model equations are detailed in de Francqueville et al. (2020b). For simplicity reasons and in order to reduce the number of adjustable parameters, the normal and tangent behaviors have been assumed similar. Therefore, the cohesive-zone model is defined by three independent parameters only, the initial stiffness $K$, the critical stress $T^{c}$ and the displacement to failure $\delta^{f}$. The local damage, characterizing the matrix debonding, is defined by the separation factor $\lambda$ defined as, $\lambda=$ $\sqrt{\left(\left\langle\delta_{n}\right\rangle / \delta^{f}\right)^{2}+\left(\delta_{t} / \delta^{f}\right)^{2}}$ with $\delta_{n}$ and $\delta_{t}$ the normal and tangent displacements of the interface (symbol $\langle\cdot\rangle$ stands for Macaulay brackets allowing separating the effect of compression or opening on the interface behavior).

A viscous regularization, such as proposed by Gao and Bower (2004), has been implemented to improve numerical convergence without modifying the results (de Francqueville et al., 2020a). The cohesive-zone elements have been implemented in the finite element code by writing a *UEL subroutine designed for quadratic prismatic 12-nodes elements. The elements, of initial null thickness, are introduced by duplication of every node at the surface of the fillers. A last difficulty stands in the fact that in order to visualize quantity related to these elements, dummy quadratic triangle membrane elements (M3D6) of close to zero stiffness had to be added. The later elements have been used to display the separation factor $\lambda$. Finally, the considered microstructures contained more than 1.3 million elements and were run on ten cluster nodes (2.5 GHz Intel Xeon E5-2640 CPU, 64 GB RAM).

\subsection{Linear behavior}

Since no damage has occurred yet at infinitesimal strain, knowing the linear elastic behavior of the fillers and the matrix, a simple option is to use a mean field micromechanics model to estimate the Young moduli of the composites. It has been shown in (Ghossein and Lévesque, 2012; Gusev, 2016; de Francqueville et al., 2019) that the generalized self-consistent (GSC) model (Christensen and Lo, 1979; Christensen, 1990) defined for spherical particles, provides with the same results as a finite elements numerical homogenization approach. The Young modulus of the matrix filled with $20 \%$ of aluminium powder is estimated to $0.57 \mathrm{MPa}$ with the GSC model using the standard aluminium properties $E=69 \mathrm{GPa}$ and $\nu=0.346$ (Ashby, 1981).

Estimating the propellants properties with the GSC model provides with satisfactory values of $9.8 \mathrm{MPa}$ and $3.8 \mathrm{MPa}$ for material AP_al and $\mathrm{RDX}$ respectively. Actually, despite their polyhedral shapes, the particles shape ratios are close to one, which can explain why the GSC model with spherical particle assumption works well. It seems that the faceted

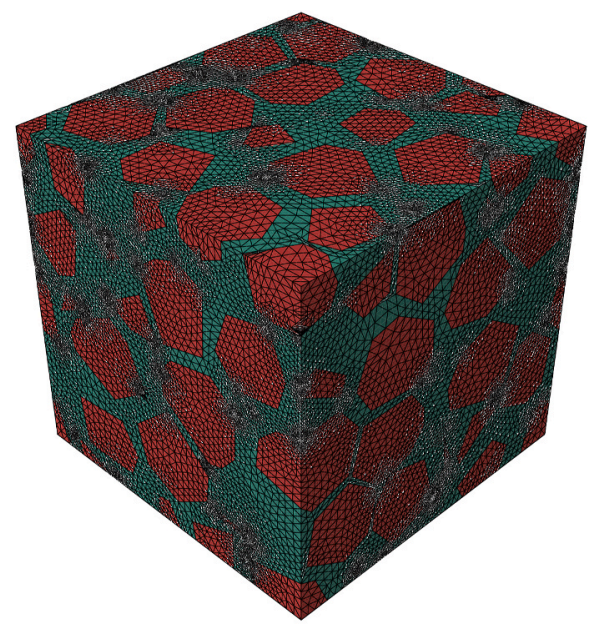

(b)

Fig. 7. Representative periodic microstructures of $1 \mathrm{~mm}^{3}$ volume with 64 monosized (a) spheres and (b) polyhedral particles filling $55 \%$ of the cube volume. 

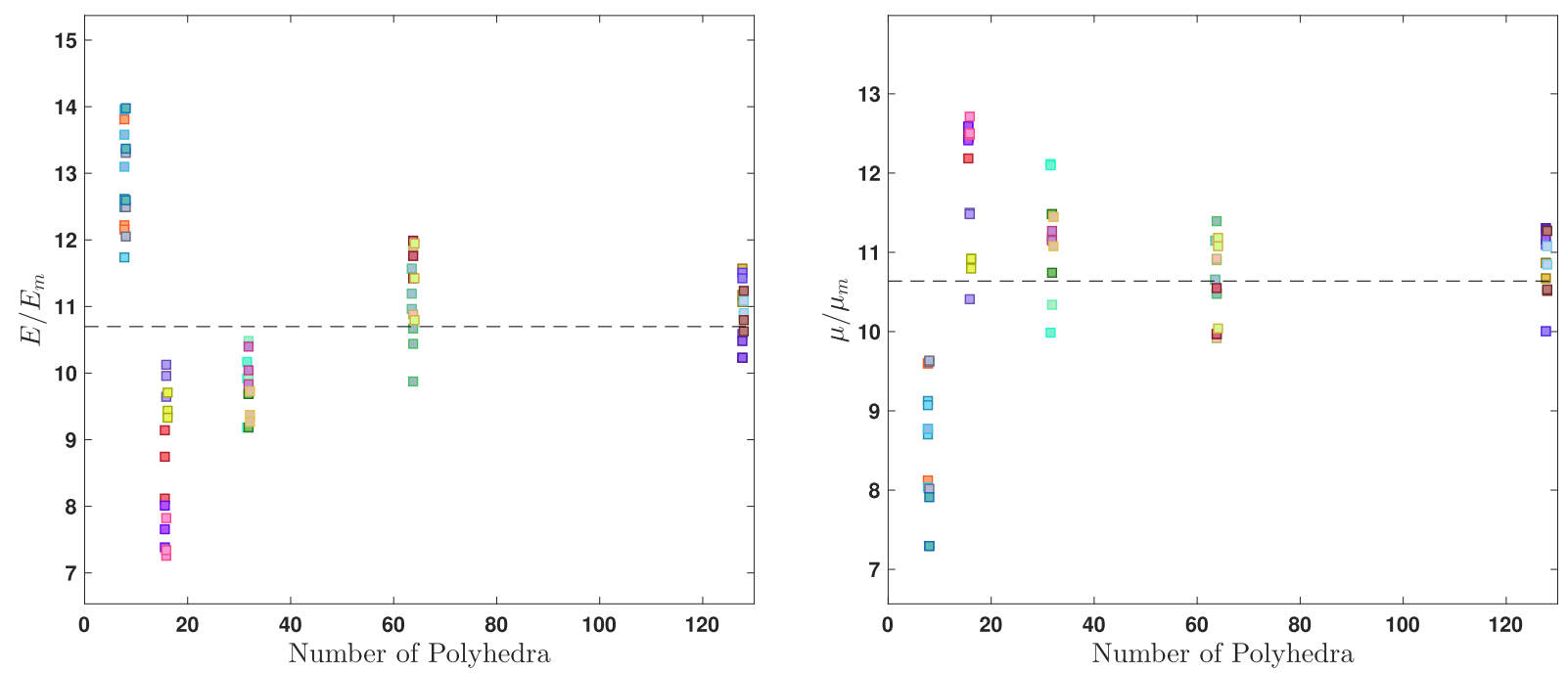

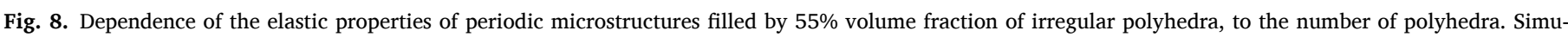

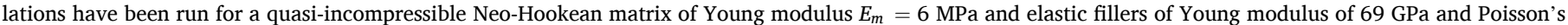
ratio 0.25 .

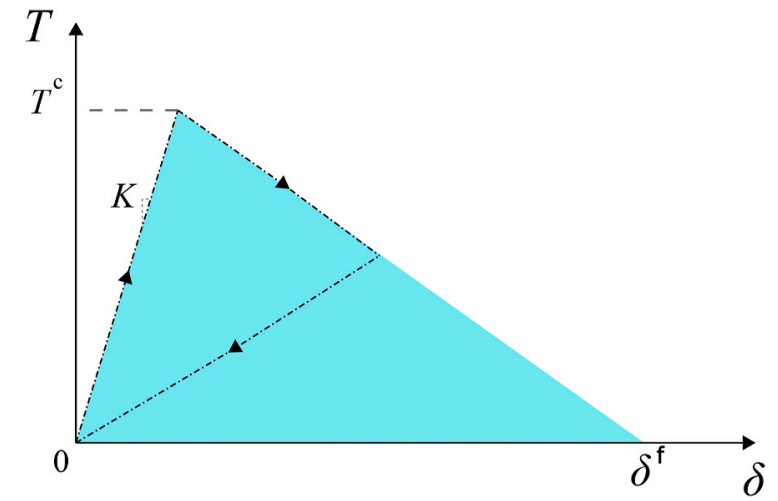

Fig. 9. Behavior of a cohesive-zone element for a purely normal (or purely tangent) displacement. Parameters $K, T^{c}$ and $\delta^{f}$ designate the initial stiffness, the critical stress and the displacement to failure of the traction-separation law.

aspects of the fillers have not a significant impact at small strain. However, for material AP, a deceiving theoretical value of $3.5 \mathrm{MPa}$ is obtained, demanding a richer modeling analysis to predict the composite linear response.

In order to better understand the case of material AP, finite element calculations were carried out on microstructures filled with particles. Moreover, in order to continue the comparison with the GSC model, spherical particles were chosen. The constitutive phases are assumed elastic with perfect adhesion between the matrix and the fillers. Six microstructures with well randomly dispersed particles were first considered and submitted to uniaxial tensile tests according to the three principal axis of the cubic cells. An average Young modulus of $E=3.9$ $\mathrm{MPa}$ and a standard deviation of $0.29 \mathrm{MPa}$ have been obtained, consistent with the GSC model estimate, which did not help understanding the excessively high Young modulus value measured experimentally for material AP. Note that at $55 \%$ volume fraction of fillers, it is not obvious to generate microstructures with well dispersed particles and a relatively large number of microstructures presenting some kind of order have been discarded. During this process, two microstructures stood out, presenting a rather good isotropy with questionable randomness when evaluating the nearest neighbor distribution function (de Francqueville et al., 2019). Actually, particle percolation paths or particle clustering were suspected. Running finite element simulations
Table 2

Material parameters for the constitutive phases.

\begin{tabular}{ll}
\hline Matrix & Ogden hyperelasticity constants \\
\hline Inert matrix & $\mu=0.11 \mathrm{MPa}, \alpha=2.25$ \\
Inert matrix + aluminium & $\mu=0.19 \mathrm{MPa}, \alpha=2.25$ \\
\hline Fillers & Linear elasticity \\
\hline RDX & $E=16 \mathrm{GPa}, \nu=0.25$ \\
ammonium perchlorate & $E=19.5 \mathrm{GPa}, \nu=0.25$ \\
\hline
\end{tabular}

with material AP constitutive phase properties on these microstructures, has resulted in an average Young modulus of $E=8.3 \mathrm{MPa}$ (with a standard deviation of 1.2 MPa), significantly larger than for the other microstructures. While this result still falls short of the actual Young modulus of $18.5 \mathrm{MPa}$ of material $\mathrm{AP}$, it provides with a plausible explanation for the enhanced properties shown by material AP: poor particles dispersion resulting in percolation and/or clustering rises dramatically the material stiffness.

\subsection{Account for filler/matrix damage}

In order to deal with the large strain, simulations were run with the microstructures filled with polyhedra with an account of the matrix/ filler debonding through the cohesive-zone elements at the filler interfaces. The plain matrix mechanical behavior is described by the Ogden hyperelastic law (Fig. 1a). The matrix filled with aluminium particles is defined by the same law with a shear modulus enhanced to $\mu=0.19 \mathrm{MPa}$ in order to reach $0.57 \mathrm{MPa}$ for the Young modulus consistent with the value predicted by the GSC model. The mechanical behaviors of the matrices and fillers are reminded in Table 2.

Simulations are explored for a first set of cohesive-zone parameters (set 1 ) listed in Table 3 . Note that the energy $\Gamma$ is listed in Table 3 for

Table 3

Cohesive-zone model parameters for the interface behavior.

\begin{tabular}{lllll}
\hline & $K(\mathrm{MPa} / \mathrm{mm})$ & $T^{c}(\mathrm{MPa})$ & $\delta^{f}(\mathrm{~mm})$ & $\Gamma(\mathrm{MPa} . \mathrm{mm})$ \\
\hline Set 1 & 30 & 0.050 & 0.04 & 0.001 \\
Set 2 & 70 & 0.02 & 0.005 & 0.00005 \\
Set 3 & 6 & 0.015 & 0.02 & 0.00015 \\
\hline
\end{tabular}




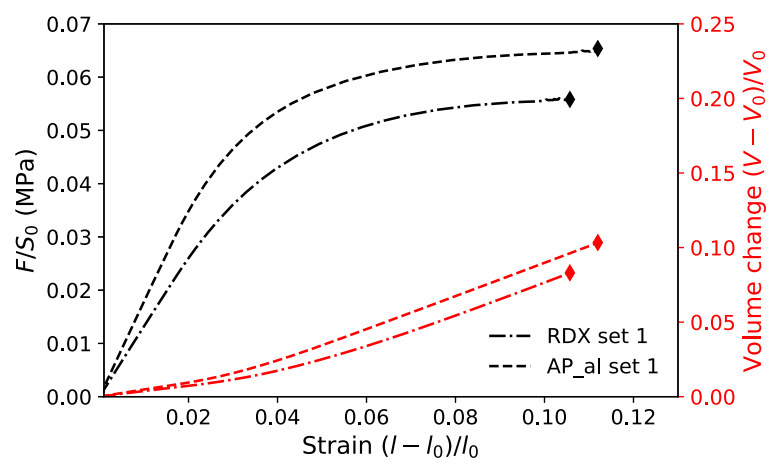

(a)

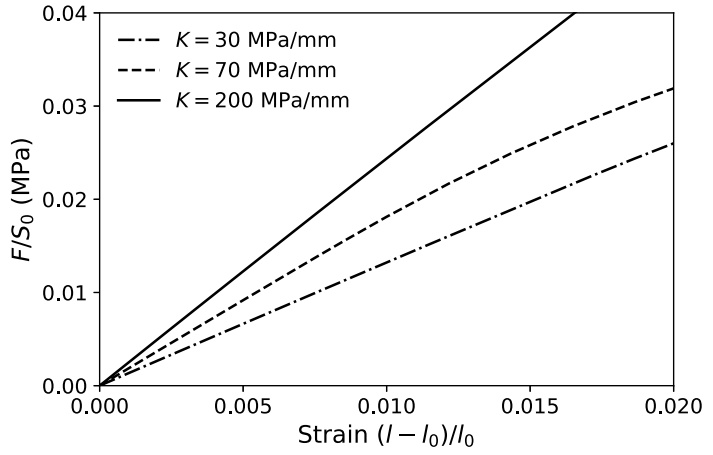

(b)

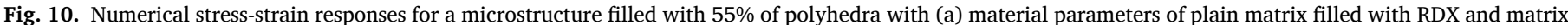

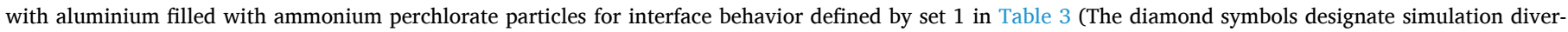
gence) - (b) material parameters of plain matrix filled with RDX with several filler/matrix interface stiffness $K$.

discussion only, since it depends on the other parameters according to the relation $\Gamma=T^{c} . \delta^{f} / 2$. The cases of the inert matrix with RDX fillers as well as the matrix with aluminum filled with ammonium perchlorate are run. Fig. 10a shows the numerical macroscopic stress-strain responses obtained for both materials. It is interesting to note that while the quantitative comparison with the experimental data is not satisfactory, the shape of the stress-strain response of the material filled with ammonium perchlorate particles is in agreement with the behavior displayed by the actual AP_al (Fig. 1b). The lack of possible quantitative comparison is due to the relatively small value of $K$. Unfortunately simulations with high values of $K$ drove to undesired early calculations divergence, and had to be given up. Nonetheless, the impact of the value of $K$ on the initial linear part of the numerical stress-strain response is displayed in Fig. 10b. As a consequence, only qualitative comparisons with the actual materials are proposed. They can provide insightful comparisons between the mechanical behaviors at the interface, and the local damage and the resulting macroscopic behaviors. When looking at the local damage in the microstructure characterized by the separation factor $\lambda$, the damage develops across the whole microstructure evenly but slowly and when simulations diverge at about $12 \%$ of strain, none of the CZM elements has reached complete separation of $\lambda=1$ (Fig. 11). This result is consistent with the presence of fibrils witnessed around the particles in material AP_al in Fig. 4.

One notes that the enhanced stiffness of the matrix mixed with aluminium particles results in a stiffer linear stress-strain response but the difference between RDX and AP_al is significantly larger. Actually, it is possible, as shown in Fig. 10b, that the difference in the linear stress-

\section{Separation factor $\lambda$}
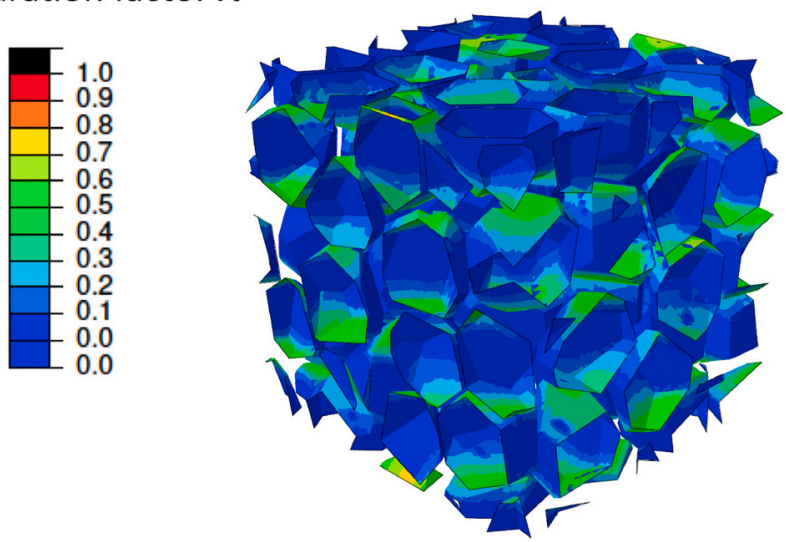

Fig. 11. Local damage characterized by the separation factor $\lambda$ for numerical material AP_al at divergence in Fig. 10a. strain responses exhibited by the actual materials at the beginning are due to different interface stiffnesses, which would increase in order from RDX to AP_al, and to AP.

Running more simulations, one notices that the material strength is controlled by parameter $T^{c}$. Nonetheless, when $T^{c}$ is lowered and $\delta^{f}$ kept constant, the shape of the stress-strain response obtained numerically is similar as those displayed in Fig. 10a and the local damage develops as in Fig. 11. Therefore, lowering the value of $T^{c}$, while necessary, is not enough to reproduce qualitatively the behaviors of materials AP and $\mathrm{RDX}$. Since the actual material AP showed complete debonding of the matrix around the fillers (Fig. 5), a very low value of $\delta^{f}$ is chosen to simulate the case of an interface that, once damaged, evolves quickly to catastrophic failure. Fig. 12 shows the stress-strain response and the local damage evolution of the microstructure filled with $55 \%$ of fillers with constitutive behavior of plain matrix and ammonium perchlorate and cohesive-zone parameters set 2 in Table 3 . The stress-strain response displays the same trend as actual material AP with the macroscopic stress decreasing once the damage sets in after the initial linear stressstrain response. As one can read on the screenshots of the microstructure damage, complete matrix/filler separation happens very quickly

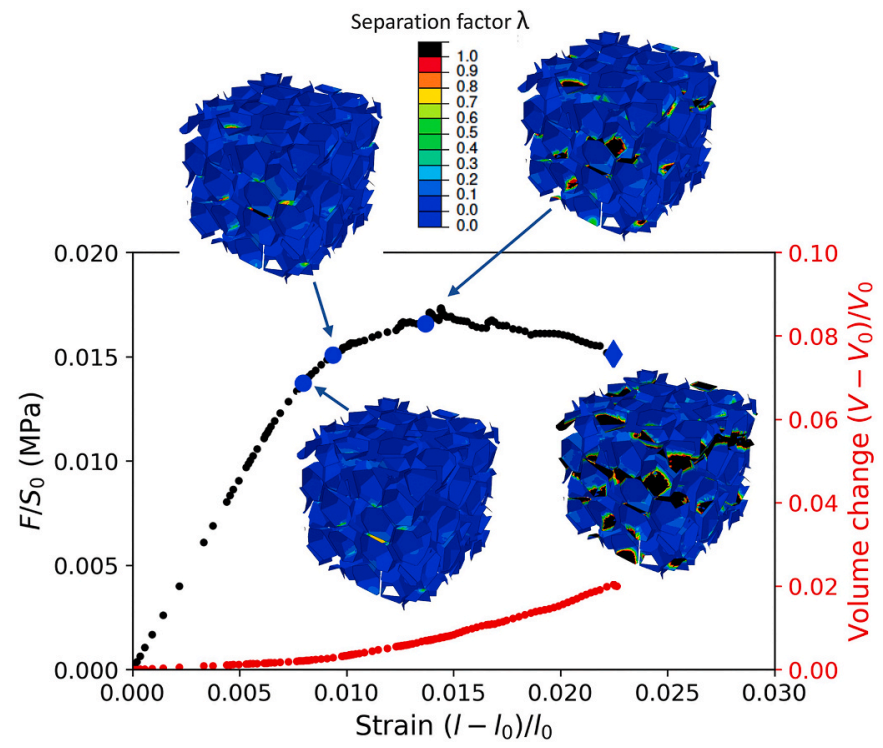

Fig. 12. Numerical stress-strain responses and local damage evolutions for a microstructure filled with $55 \%$ of polyhedra with material parameters of plain matrix filled with ammonium perchlorate particles for the interface behavior defined by set 2 in Table 3 . The diamond symbol indicates the simulation divergence. 
once damage appears on one particle. While less particles are undergoing some interfacial damage compared to the first case considered where almost every particles seem to undergo some damage (Fig. 11), when some damage happens it evolves quickly into complete failure (shown by the black color). Unfortunately, this case of microstructure damage drives to early simulation divergence.

The actual material RDX shows significantly lower initial stiffness and strength compared to material AP. Despite a different trend for the macroscopic stress-strain response, fibrils have been witnessed around the particles (Fig. 6). Therefore, simulations were run with parameters set 3 characterized by lower values of $K$ and $T^{c}$ compared to CZM parameters set 1 , and a value $\delta^{f}$ large enough to witness damaged interface without immediate complete failure, but small enough to change the behavior trend displayed in Fig. 10. Fig. 13 shows the macroscopic stress-strain response and the evolution of the local damage. As one can read on the first microstructure, damage appears evenly across the microstructure similarly to the case of parameters set 1 . While strain increases, both complete and partial matrix/filler separations are witnessed, which is consistent with images in Fig. 6 showing the presence of fibrils but also some complete separation between matrix and fillers in some area. Note that this numerical case was the last one to diverge.

To summarize, the different sets of interface behaviors that had been required to reproduce numerically the trends of the stress-strain responses displayed by the actual materials have provided with interesting insight on the material interface properties. Material AP_al shows a strong and tough interface with large critical strength and separation at break, material AP interface breaks early after the damage appearance due to a low separation at break limit, and material RDX displays an intermediate interface, with low strength favoring the damage to set in early, that resists to complete break thanks to a large separation at failure.

\section{Conclusion}

The development of new propellants is constrained by the fact that these materials have to reach given strength and toughness requirements. Upon mechanical loadings, propellants soften due to matrix debonding at the filler interface. Three propellant-like materials have been made and submitted to monotonic uniaxial tensile tests, while the damage was monitored at the macroscopic scale by measuring the increase of sample volumes, and at the microscopic scale by imaging their surfaces upon stretching with a scanning electron microscope. Very different stress-strain responses were observed resulting from different types of matrix/fillers debonding signatures, from showing a lot of interfacial fibrils to none. In order to better understand the impact of the matrix to filler adhesion properties on the damage appearance and evolution, and the resulting macroscopic stress-strain response for these materials, finite element analyses have been carried out. For this purpose, an original microstructure generation tool was used to create periodic representative element volumes of a soft matrix highly filled with non-regular polyhedral particles randomly dispersed. The matrix/filler debonding has been taken into account thanks to a simple cohesive-zone model at the matrix/filler interface. Qualitative comparisons between the simulations and the experiments have given valuable informations on the relationship between the shape of the macroscopic stress-strain responses and the mechanical behavior at the filler interfaces. Material AP_al presenting a good strength but breaking early is likely to present a high strength and tough interface. Material AP presenting an early and steady downturn of the macroscopic stress and no fibrils around the particles at the microscopic scale is likely to present an interface with small separation failure driving to complete matrix debonding rapidly after damage sets in. Finally, material RDX showing low macroscopic strength and large strain at break is likely to present an interface with low critical strength favoring the spread of damage and an intermediate separation to failure limiting stress concentration and complete debonding.

In order to improve the simulations that have been proposed and reach quantitative comparisons between the materials of interest and similar numerical simulations several paths may be envisioned. In order to limit the convergence problems encountered with large values of $K$ and provide with more versatility for the CZM parameters, one may differentiate the tangent from the normal damage, or use richer CZM constitutive equations such as (Park et al., 2009). However, such a choice will increase the number of phenomenological parameters rendering more difficult the physical interpretation. Another possibility is to introduce a layer of interphase around the particles. Although this also will increase the number of parameters since the behavior of the interphase will have to be guessed, it will help explore the impact of particle percolation or clustering since the presence of a continuous interphase seems to enhance significantly the mechanical properties of

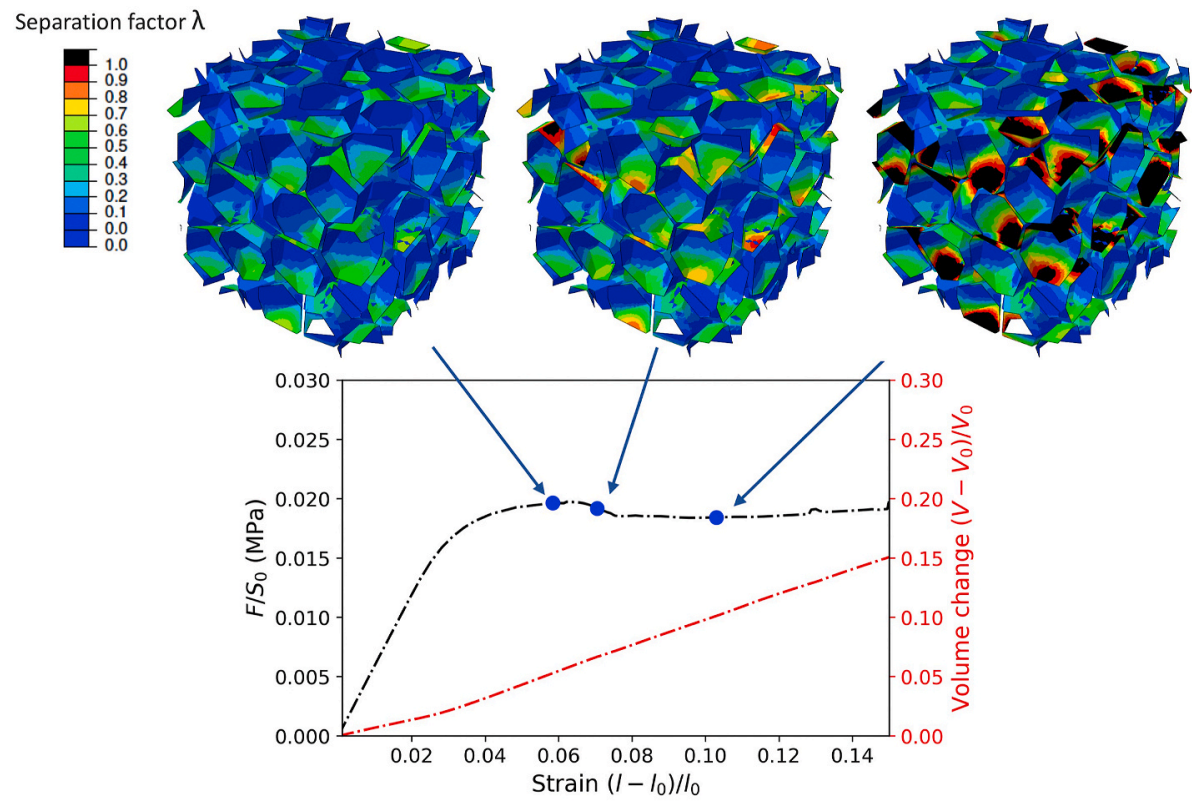

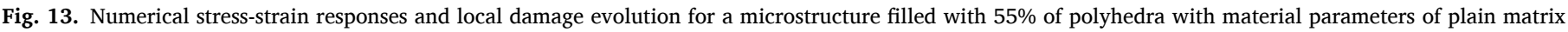
filled with RDX particles for interface behavior defined by set 3 in Table 3 . 
highly filled microstructures (Toulemonde et al., 2016).

\section{Declaration of competing interest}

The authors declare that they have no known competing financial interests or personal relationships that could have appeared to influence the work reported in this paper.

\section{Acknowledgements}

This work was supported by the ANR (France) under contract number ANR-10-EQPX- 37, and the authors thank the Délégation Générale de l'Armement (DGA) and ArianeGroup, Vert-le-Petit (France), for their financial supports.

\section{References}

Abaqus/Standard, 2018. Dassault Systèmes Simulia Corporation. Johnston, RI, USA. Ashby, M.F., Jones, D.R.H., 1981. Matériaux. Dunod.

Barua, A., Horie, Y., Zhou, M., 2012. Microstructural level response of hmX-estane polymer-bonded explosive under effects of transient stress waves. Proc. Roy. Soc. Lond. A 468, 3725-3744.

Christensen, R.M., 1990. A critical evaluation for a class of micro-mechanics models. J. Mech. Phys. Solid. 38 (3), 379-404.

Christensen, R.M., Lo, K.H., 1979. Solutions for effective shear properties in three phase sphere and cylinder models. J. Mech. Phys. Solid. 27 (4), 315-330.

Cornwell, L.R., Schapery, R.A., 1975. SEM study of microcracking in strained solid propellant. Metallography 8 (5), 445-452.

Dai, K., Lu, B., Chen, P., Chen, J., 2019. Modelling microstructural deformation and the failure process of plastic bonded explosives using cohesive zone model. Materials 12 3661.

de Francqueville, F., Gilormini, P., Diani, J., 2019. Representative volume elements for the simulation of isotropic composites highly filled with monosized spheres. Int. J. Solid Struct. 158, 277-286.

de Francqueville, F., Gilormini, P., Diani, J., Vandenbroucke, A., 2020a. Relationship between local damage and macroscopic response of soft materials highly reinforced by monodispersed particles. Mech. Mater. 146, 103408.

de Francqueville, F., Gilormini, P., Diani, J., Vandenbroucke, A., 2020b. Comparison of the finite strain macroscopic behavior and local damage of a soft matrix highly reinforced by spherical or polyhedral particles. Eur. J. Mech. Solids A 84, 104070.

Farris, R.J., 1964. Dilatation of granular filled elastomers under high rates of strain. J. Appl. Polym. Sci. 8 (1), 25-35.

Fritzen, F., Böhlke, T., Schnack, E., 2009. Periodic three-dimensional mesh generation for crystalline aggregates based on Voronoi tessellations. Comput. Mater. 43 (5), 701-713.

Gao, Y.F., Bower, A.F., 2004. A simple technique for avoiding convergence problems in finite element simulations of crack nucleation and growth on cohesive interfaces. Model. Simulat. Mater. Sci. Eng. 12, 453-463.

Ghossein, E., Lévesque, M., 2012. A fully automated numerical tool for a comprehensive validation of homogenization models and its application to spherical particles reinforced composites. Int. J. Solid Struct. 49 (11-12), 1387-1398.

Gilormini, P., Toulemonde, P.A., Diani, J., Gardère, A., 2017. Stress-strain response and volume change of a highly filled rubbery composite: experimental measurements and numerical simulations. Mech. Mater. 111, 57-65.
Gusev, A.A., 1997. Representative volume element size for elastic composites : a numerical study. J. Mech. Phys. Solid. 45 (9), 1449-1459.

Gusev, A.A., 2016. Controlled accuracy finite element estimates for the effective stiffness of composites with spherical inclusions. Int. J. Solid Struct. 80, 227-236.

Heuillet, P., 1992. Caractérisation de l'endommagement des propergols solides composites. Ph.D. thesis, Compiègne.

Huang, J., Lu, S., Xie, F., Liu, W., Xiao, C., Zhang, J., Sun, T., 2020. Finite element analysis of synergetic deformation in precision cutting of polymer bonded explosive. Mater. Des. 168, 108471.

Hudson, R.J., Zioupos, P., Gill, P.P., 2012. Investigating the mechanical properties of rdx crystals using nano-indentation. Propellants, Explos. Pyrotech. 37 (2), 191-197.

Inglis, H.M., Geubelle, P.H., Matouš, K., Tan, H., Huang, Y., 2007. Cohesive modeling of dewetting in particulate composites: micromechanics vs. multiscale finite element analysis. Mech. Mater. 39 (6), 580-595.

Keyhani, A., Kim, S., Horie, Y., Zhou, M., 2019. Energy dissipation in polymer-bonded explosives with various levels of constituent plasticity and internal friction. Comput. Mater. Sci. 159, 136-149.

Kumar, M., Kovacic, S.M., Kuo, K.K., 1981. Flame propagation into the crack of a solid propellant cracks. AIAA J. 19 (5), 610-618.

Li, G., Wang, Y., Jiang, A., Yang, M., Li, J., 2018. Micromechanical investigation of debonding processes in composite solid propellants. Propellants, Explos. Pyrotech. 43, 642-649.

Lubachevsky, B.D., Stillinger, F.H., 1990. Geometric properties of random disk packings. J. Stat. Phys. 60 (5-6), 561-583.

Lucca, D.A., Klopfstein, M.J., Mejia, O.R., Rossettini, L., DeLuca, L.T., 2006. Investigation of ammonium perchlorate by nanoindentation. Mater. Sci. Technol. 22 (4), 396-401.

Matouš, K., Geubelle, P.H., 2006. Multiscale modelling of particle debonding in reinforced elastomers subjected to finite deformations. Int. J. Numer. Methods Eng. 65 (2), 190-223.

Oberth, A.E., Bruenner, R.S., 1965. Tear phenomena around solid inclusions in castable elastomers. Trans. Soc. Rheol. 9 (2), 165-185.

Ogden, R.W., 1972. Large deformation isotropic elasticity - on the correlation of theory and experiment for incompressible rubberlike solids. Proc. Roy. Soc. Lond. 326, $565-584$.

Park, K., Paulino, G.H., Roesler, J.R., 2009. A unified potential-based cohesive model of mixed-mode fracture. J. Mech. Phys. Solid. 57, 891-908.

Quey, R., Dawson, P.R., Barbe, F., 2011. Large-scale 3d random polycrystals for the finite element method: generation, meshing and remeshing. Comput. Methods Appl. Mech. Eng. 200, 1729-1745.

Schöberl, J., 1997. NETGEN an advancing front 2D/3D-mesh generator based on abstract rules. Comput. Visual Sci. 1 (1), 41-52.

Torquato, S., al, 2006. http://chemlabs.princeton.edu/torquato/links-and-codes/spheres -packings-registration/.

Toulemonde, P.A., Diani, J., Gilormini, P., Lacroix, G., Desgardin, N., 2016. Roles of the interphase stiffness and percolation on the behavior of solid propellants. Propellants, Explos. Pyrotech. 41, 978-986.

Tvergaard, V., Hutchinson, J.W., 1993. The influence of plasticity on mixed mode interface toughness. J. Mech. Phys. Solid. 41 (6), 1119-1135.

Weng, L., Fan, T., Wen, M., Shen, Y., 2019. Three-dimensional multi-particle FE model and effects of interface damage, particle size and morphology on tensile behavior of particle reinforced composites. Compos. Struct. 209, 590-605.

Williams, J.J., Segurado, J., Llorca, J., Chawla, N., 2012. Three dimensional (3d) microstructure-based modeling of interfacial decohesion in particle reinforced metal matrix composites. Mater. Sci. Zng. A. 55, 113-118.

Zhong, A., Knauss, W.G., 1997. Analysis of interfacial failure in particle-filled elastomers. J. Eng. Mater. Technol. 119 (3), 198-204.

Zhong, A., Knauss, W.G., 2000. Effects of particle interaction and size variation on damage evolution in filled elastomers. Mech. Comp. Struct. Mater. 7 (1), 35-53. 